\title{
Experimental Study of ABS Material Shrinkage and Deformation Based on Fused Deposition Modeling
}

\author{
Yaodong $\mathrm{XU}^{1, \text { a }}$ \\ ${ }^{1}$ Department of Manufacturing Engineering, Shanghai University of Engineering \\ Science,Shanghai,200437,China \\ asodarbiscuit@163.com
}

\begin{abstract}
The CAD model can be directly converted into physical model by rapid prototyping, which provides more convenient means for concurrent design in physical verification. However, the deformation of the material shrinkage can cause large deviation of the molding size in CAD model transforming to physical model. In order to ensure the reasonable assembly of the rapid prototyping part, the forming experiment of ABS material was carried out by the principle of FDM. Through regression analysis, a linear relationship with the molding shrinkage and model size was found .Meanwhile, deforming mechanism of prototyping parts was analyzed.The rationality of assembly and quality of print parts can be ensured by these law of pre-process.
\end{abstract}

\section{Introduction}

The quality of the product is $90 \%$ depending on the quality of the design, and good design must consider the process of concurrent design, that is, from product design to manufacturing, assembly and then to design to finish the closed-loop process. The emergence of rapid prototyping technology makes the concept design into the physical model to be a reality, with the rapid prototyping technology printing model instead of the actual processing parts for assembly, structure process analysis, functional testing, motion simulation and verification, that can improve the reliability and quality of the design, shorten the design development cycle, so that the product can more quickly put into market[1].

Rapid prototyping technology can directly manufacture equipment, but taking into account the problem of material shrinkage, rapid prototyping prototype parts dimensional tolerances are inconsistent with general mechanical parts dimensional tolerances.Because rapid prototyping is based on $\mathrm{CAD}$ model, and $\mathrm{CAD}$ model design in the size and tolerance are the size and tolerance of mechanical design, if the CAD model is directly printed from the mechanical design, it will produce printed parts that can not be assembled, or assembly parts can not be disassembled. In order to make the rapid prototyping to reflect the assembly relationship, it is necessary to make a reasonable change to the size of the parts, or reasonable scaling of the parts. In this paper, we have made the shrinkage and deformation experiments of the prototype parts. Through the data analysis, we find the law of shrinkage and deformation of the prototype, so as to ensure the rapid prototyping parts

* Corresponding author: sodarbiscuit@163.com 
assembly, and to lay the foundation for the process analysis and performance test of the whole prototype later on.

At present, the research of rapid prototyping technology in domestic and abroad mainly involves the forming quality, forming process, forming new material. In the development of new material, the research focused on the low shrinkage rate, the strength and toughness, and the low cost of the material.In molding process parameters optimization, such as molding temperature, thickness, printing speed and other factors on the influence of the size of the prototype, a large number of experiments were carried out[2]. However, the study of the previous research was more aimed at the quality of a single prototype, but if the prototyping part were to be assembled, it should not only consider the quality, but also the size of it to meet the requirements of assembly, and the size of contraction in the case of the model size change. At present,3D printing assembly is realized by two kinds of processes.One is to design parameters of the model, to make different scaling, and then print. The second is to use 3D printer system software to scale the model.

Based on the experimental study, combined with regression analysis method, the paper takes the principle of FDM as an example, to find out a linear relationship and empirical formula verified by data, in the shrinkage deformation, the model size, material filling rate and so on.

\section{Molding Shrinkage Test}

In the experiment, the ABS wire material was used to FDM. The 3D printer was GIA, and the processing software was Aurora. Printing process parameters were double nozzle print, print temperature 230 degrees, layer thickness $0.2 \mathrm{~mm}$, shell print 3 layer, the contour line width $0.5 \mathrm{~mm}$, filling line width $0.5 \mathrm{~mm}$, filling interval $2.5 \mathrm{~mm}$.

Select the shaft of $\phi 12 \mathrm{~mm}$ and the handle hole of $\phi 12 \mathrm{~mm}$ to assembly in diameter. The shaft scaled in diameter by the proportion of $0.736 \mathrm{zoom}$. In order to ensure that the handle and shaft can be matched,experiment was carried out according to the different proportion of handle scaling, the experimental data are shown in table 1.

TABLE1. THE MOLDING SHRINKAGE TEST DATA

\begin{tabular}{|c|c|c|c|c|c|}
\hline & Shaft & \multicolumn{4}{|c|}{ Hole } \\
\hline Scaling & 0.736 & 0.78 & 0.742 & 0.73 & 0.69 \\
\hline $\begin{array}{c}\text { Theoretical } \\
\text { Dimension }\end{array}$ & 8.832 & 9.36 & 8.904 & 8.76 & 8.28 \\
\hline Actual Size & 8.76 & 9.18 & 8.75 & 8.62 & 8.16 \\
\hline Shrinkage Ratio & $0.8 \%$ & $1.9 \%$ & $1.7 \%$ & $1.6 \%$ & $1.4 \%$ \\
\hline
\end{tabular}

Regression Analysis.According to table 1, with the change of the proportion of the hole scaling, the proportion of molding shrinkage is also changing, and there is a positive correlation. Through linear regression analysis, and correlation calculation [3], the scaling of the printing model $x_{i}$ is linear with the proportion of the shrinkage of the model $y_{i}$. Correlation calculation data are shown in table $2, r_{x y}$ is the correlation coefficient as formula 1 . 


$$
r_{x y}=\frac{\sum_{i=1}^{N}\left(x_{i}-\bar{x}\right)\left(y_{i}-\bar{y}\right)}{\sqrt{\sum_{i=1}^{N}\left(x_{i}-\bar{x}\right)^{2}} \sqrt{\sum_{i=1}^{N}\left(y_{i}-\bar{y}\right)^{2}}}
$$

TABLE2.RELATIVITY CALCULATING DATA

\begin{tabular}{|c|c|c|c|c|c|c|c|}
\hline $\begin{array}{c}\text { Measurement } \\
\text { number } i\end{array}$ & $\begin{array}{c}\text { Scaling } \\
x_{i}\end{array}$ & $\begin{array}{c}\text { Shrinkage } \\
\text { ratio } y_{i} / \%\end{array}$ & $x_{i}^{2}$ & $x_{i} \cdot y_{i}$ & $x_{i}-\bar{x}$ & $y_{i}-\bar{y}$ & $\left(x_{i}-\bar{x}\right)\left(y_{i}-\bar{y}\right.$ \\
\hline 1 & 0.69 & 1.4 & 0.4761 & 0.966 & -0.0433 & -0.2333 & 0.0101 \\
\hline 2 & 0.73 & 1.6 & 0.5329 & 1.168 & -0.0033 & -0.0333 & 0.0001 \\
\hline 3 & 0.78 & 1.9 & 0.6084 & 1.482 & 0.0467 & 0.2667 & 0.0124 \\
\hline average value & $\bar{x}=0.7333$ & $\bar{y}=1.6333$ & & & & & \\
\hline$\Sigma$ & & & 1.6174 & 3.616 & & & 0.0226 \\
\hline
\end{tabular}

The fitting curve of molding shrinkage ratio and the scaling of the model is shown in Figure.1.Equation of linear regression $y=5.5738 x-2.4541$.

According to linear regression equation $y=5.5738 x-2.4541$,molding shrinkage ratio $y$ is about $1.7 \%$ when molding shrinkage ratio $x$ is 0.742 . The actual shrinkage rate of the model is also $1.7 \%$ after printing the model according to the scale of 0.742 as shown in
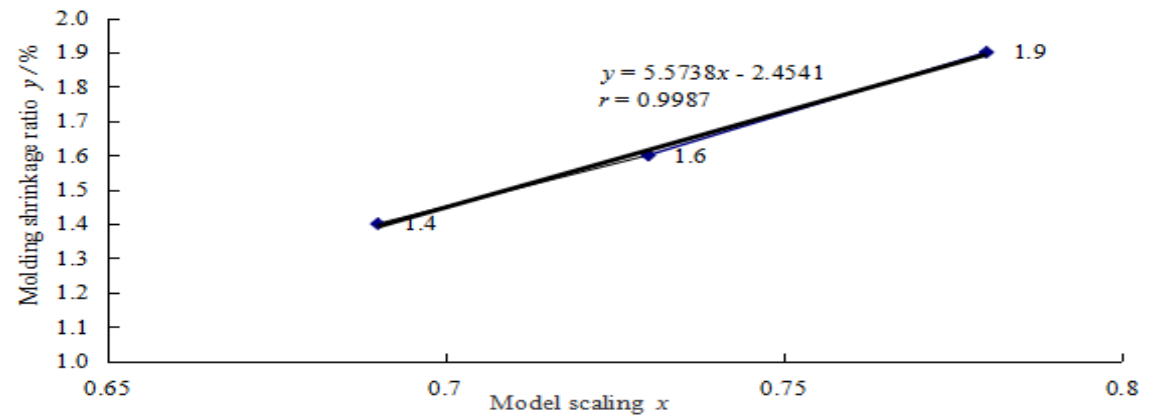

Fig.1. The fitting curve of the molding shrinkage ratio and the model scaling

Table 1. The correctness of the linear relationship between the proportion of molding shrinkage and the scaling of the model is verified.

Empirical Formula.Through analysis, material shrinkage is related with filling rate of model material besides ABS itself material shrinkage[4].Layer thickness, the number of layers and the grid gap in print will determine the material filling rate.With the printing model smaller,the filling rate of the material will be greater if layer thickness, layer number of shell and the grid gap are unchanged, so that the prototyping parts will become more solid.On the contrary, the larger the printing model, the larger the hollow area is and the smaller the filling rate is. Through the analysis of experimental verification, the molding shrinkage ratio is a, the shrinkage of the material is b, and the filling rate of the material is c, then the empirical formula is got as formula 2. 
The filling rate of actual material can be calculated by the ratio of ABS material consumption and volume of theoretical model.For example,ABS material mass used in the print handle is 2.2 gram. Handle model volume is $2.1586 \times 10^{3} \mathrm{~mm}^{3}$ calculated by PROE software mass analysis.

According to the density of ABS material $\rho=1.05 \mathrm{~g} / \mathrm{cm}^{3}$, the actual handle volume of material is got as formula 3 .

$$
V=\frac{m}{\rho}=\frac{2.2 \times 10^{3}}{1.05}=2095.2 \mathrm{~mm}^{3}
$$

According to the empirical formula $a=\frac{b}{c}$, handle molding shrinkage ratio $a=\frac{b}{c}=\frac{b}{V / V_{0}}=\frac{0.8 \%}{2095.2 / 2158.6}=0.866 \%$,meanwhile shrinkage rate of ABS material is $0.8 \%$.As known $0.742 \times(1-0.866 \%) \approx 0.736$, the $\mathrm{H} / \mathrm{h}$ tolerance fit is achieved as the dimension of handle scaled by 0.742 is equal to the print axis with the scale of 0.736 after the contraction.

\section{Deformation Mechanism Analysis}

Cracking. The main factors that affect the cracking are printing temperature, printing speed, number of layers, cooling temperature, etc. As print temperature is low, wire material is not sticky enough to connect and as the scanning speed of nozzle, or called the speed of space movement is higher than that of wire extruding, it can cause wire material stretched or broken as well as vibration of machine owing to fast speed,resulting in model cracking[5]. In addition, the interval between layers is determined by the number of layers, and with the number of layers less, the interval is larger, causing the adhesion of wire material is not good, so the adhesion between layers can be improved by increasing the number of layers. Otherwise,cracking is due to the uneven cooling rate in the upper and lower layer contraction, which can be solved by the constant temperature warehouse, or the model shell extraction processing to improve the heat dissipation area, reducing cracking occurs[6].

Warping. The warping is mainly related to the adhesion of the wire material to the tray, the model cracking, the filling rate of the material and the position of the model. It can generally avoid warping by less gap between the nozzle and the tray, less cracking, less material filling rate or more support supplied in printing, and reasonable model position to have less printing section.

Collapse. The factors that affect the collapse include the printing temperature, material filling rate, etc. The collapse can be reduced by the appropriate reduction of the printing temperature, increasing the filling rate of the material or increasing the support.

Roundness Error. The factors that affect the roundness error are the principle error of STL mesh[7], the bonding error between the printing layers, and the filling rate of the material, etc. STL is a model with triangle mesh, and mesh density decides the chord size which may reduce the principle error through reducing the size of it. In addition, model is 
printed by layers, the former layer into the next layer will produce joints, causing roundness error. The errors caused by the above two factors are of the principle error and can not be eliminated. Through several prototyping tests,it is concluded that the roundness error is small when print model axis perpendicular to the print level, at this time the error of the model is mainly caused by the principle error. When the axis of the printing model is parallel to the plane, the model roundness error is mainly related to the filling rate of the material.

\section{Summary}

Using rapid prototyping technology to print the CAD model into the physical model, it will appear assembly question because of the shrinkage of the material.Through experiment of shaft and hole assembly by FDM prototyping with ABS material, a linear relationship between the model scaling and the shrinkage of the prototype was found by regression fitting analysis of test data .

Applying the regression equation, the reasonable scaling ratio of the hole and axis can be predicted to meet the requirements of assembly. Through the experimental analysis, the empirical formula about molding shrinkage ratio, the material shrinkage rate and material filling rate is obtained, which can provide the necessary reference for model scaling in 3D printing assembly.

\section{Acknowledgement}

In this paper, the research was sponsored by Shanghai Municipal Education Commission Key Project(Project No. BJYXX13YYYJ00) and Shanghai University Experimental Technology Team Construction Plan(Project No. A2-8950-13-0706).

\section{References}

1. Nan Zhang and Fei Li.The development and application of 3D printing technology for future product design[J].Machine Design,2013,30(7):97-99.

2. Ronghua Ni.Study on Rapid Prototyping accuracy of fused deposition and numerical simulation of its forming process[D].Shandong:ShanDong University.2013.

3. Yaodong Xu and Wei Zheng.Carbon Content Confirmation of Annealed Carbon Steel by Hardness Measurement[J].Research and Exploration in Laboratory,2012,31(1):50-52.

4. Xuefang Chen and Yiping Zhang.Research about the ways to control the error of FDM forming workpiece[J].Modern Manufacturing Engineering,2011(11):39-41.

5. Anhua Peng ,Jianfeng Zhang and Jianglin Zhang.Experimental Research into the Influence of Technical Parameters on Parts Precision[J].JOURNAL OF HUAIHAI INSTITUTE OF TECHNOLOGY(NATURAL SCIENCES EDITION),2008,17(3):21-24.

6. Jianfeng Tan,Zhichao Wu and Zhongyu Quan,etc.The Applications of FDM Rapid Prototyping Technology in Animation Model Manufacturing[J].Machinery Design \& Manufacture,2014(6):233-235.

7. Yaodong Xu.Based on Reverse-design Broken Parts Fast Prototype and Quality Analysis[J].WORLD SCI-TECH R \& D,2009,31(5):864-866. 